\title{
Electronic Phase Separation in the Slightly Underdoped Iron Pnictide Superconductor $\mathrm{Ba}_{1-x} \mathrm{~K}_{x} \mathrm{Fe}_{2} \mathrm{As}_{2}$
}

\author{
J. T. Park, ${ }^{1}$ D. S. Inosov, ${ }^{1}$ Ch. Niedermayer, ${ }^{2}$ G. L. Sun, ${ }^{1}$ D. Haug, ${ }^{1}$ N. B. Christensen, ${ }^{2,3,4}$ R. Dinnebier, ${ }^{1}$ A. V. Boris, ${ }^{1}$ \\ A. J. Drew, ${ }^{5,6}$ L. Schulz, ${ }^{5}$ T. Shapoval, ${ }^{7}$ U. Wolff, ${ }^{7}$ V. Neu, ${ }^{7}$ Xiaoping Yang, ${ }^{1}$ C. T. Lin, ${ }^{1}$ B. Keimer, ${ }^{1}$ and V. Hinkov ${ }^{1, *}$ \\ ${ }^{1}$ Max-Planck-Institut für Festkörperforschung, Heisenbergstraße 1, 70569 Stuttgart, Germany \\ ${ }^{2}$ Laboratory for Neutron Scattering, ETHZ \& PSI, CH-5232 Villigen PSI, Switzerland \\ ${ }^{3}$ Materials Research Department, Ris $\phi$ National Laboratory for Sustainable Energy, Technical University of Denmark, \\ DK-4000 Roskilde, Denmark \\ ${ }^{4}$ Nano-Science Center, Niels Bohr Institute, University of Copenhagen, DK-2100 Copenhagen, Denmark \\ ${ }^{5}$ Department of Physics and Fribourg Center for Nanomaterials, University of Fribourg, \\ Chemin du Musée 3, CH-1700 Fribourg, Switzerland \\ ${ }^{6}$ Physics Department, Queen Mary, University of London, London, E1 4NS, United Kingdom \\ ${ }^{7}$ IFW Dresden, Institute for Metallic Materials, P.O. Box 270116, D-01171 Dresden, Germany
}

(Received 14 November 2008; published 20 March 2009)

\begin{abstract}
Here we present a combined study of the slightly underdoped novel pnictide superconductor $\mathrm{Ba}_{1-x} \mathrm{~K}_{x} \mathrm{Fe}_{2} \mathrm{As}_{2}$ by means of $\mathrm{x}$-ray powder diffraction, neutron scattering, muon-spin rotation $(\mu \mathrm{SR})$, and magnetic force microscopy (MFM). Static antiferromagnetic order sets in below $T_{m} \approx 70 \mathrm{~K}$ as inferred from the neutron scattering and zero-field- $\mu$ SR data. Transverse-field $\mu$ SR below $T_{c}$ shows a coexistence of magnetically ordered and nonmagnetic states, which is also confirmed by MFM imaging. We explain such coexistence by electronic phase separation into antiferromagnetic and superconductingor normal-state regions on a lateral scale of several tens of nanometers. Our findings indicate that such mesoscopic phase separation can be considered an intrinsic property of some iron pnictide superconductors.
\end{abstract}

DOI: 10.1103/PhysRevLett.102.117006

PACS numbers: 74.70.-b, 25.40.Dn, 68.37.Rt, 76.75.+i

Nearly one year after the discovery of high-temperature superconductivity in the iron arsenides [1,2], many important physical issues concerning these new materials are still discussed controversially, such as the pairing symmetry in the superconducting (SC) state [3], the dramatically different magnetic phase diagrams [4-8], and the precise nature of the antiferromagnetic spin-density-wave (SDW) ground state of the parent compound [9]. In addition, it has been reported that SC and antiferromagnetic (AFM) order are either well separated or coexist in the underdoped region of the phase diagram. More specifically, in $\mathrm{LaFeAsO}_{1-x} \mathrm{~F}_{x}$, the transition between the AFM and SC phases was reported to be first-order-like with no coexistence between the two phases [4], in $\mathrm{CeFeAsO}_{1-x} \mathrm{~F}_{x}$ the transition is more second-order-like, but still the AFM and SC domes do not overlap in the phase diagram [5], whereas the coexistence of the two phases was reported in a narrow doping range in $\mathrm{SmFeAsO}_{1-x} \mathrm{~F}_{x}[8,10]$, and in a broader range in $\mathrm{Ba}_{1-x} \mathrm{~K}_{x} \mathrm{Fe}_{2} \mathrm{As}_{2}$ (BKFA) [6,7]. However, there is no consensus yet about the nature of such coexistence-whether it is purely electronic or related to chemical homogeneity of the sample and the characteristic spatial scale of the corresponding phases. One of the reasons for such uncertainty is that many of the samples employed so far were powders.

Besides, complementary experiments on nominally the same composition were often performed on samples prepared by different labs. Thus, to overcome this uncertainty, we have performed dc susceptibility, $\mathrm{x}$-ray powder diffraction (XRPD), neutron scattering, and $\mu$ SR measurements on the same BKFA samples. In these measurements, a phase-separated coexistence between static magnetic order and nonmagnetic (either SC below $T_{c}$ or normal-state above $T_{c}$ ) regions was observed, and we suggest that this phase separation is an intrinsic property of underdoped BKFA, resulting from an electronic instability to the formation of static AFM islands surrounded by nonmagnetic regions below the AFM transition temperature. In this respect, the iron-arsenide superconductors resemble transition metal oxides, where electronic-phase-separation phenomena are common at various spatial scales [11], such as cuprates [12,13] or manganites [14]. On the other hand, as we will subsequently show, a quantitative comparison reveals essential differences between the characteristic scales of the inhomogeneities, which might affect the macroscopic physical properties of these materials.

Our single crystals of BKFA were grown using Sn as flux in a zirconia crucible sealed in a quartz ampoule filled with Ar. A mixture of $\mathrm{Ba}, \mathrm{K}, \mathrm{Fe}, \mathrm{As}$, and $\mathrm{Sn}$ in a weight ratio of BKFA:Sn $=1: 85$ was heated in a box furnace up to $850^{\circ} \mathrm{C}$ and kept constant for 2-4 hours to soak the sample in a homogeneous melt. A cooling rate of $3{ }^{\circ} \mathrm{C} / \mathrm{h}$ was then applied to decrease the temperature to $550{ }^{\circ} \mathrm{C}$, and the grown crystals were then decanted from the flux [15].

Sample characterization by resistivity (not shown) and dc-susceptibility measurements (see inset in Fig. 1) re- 


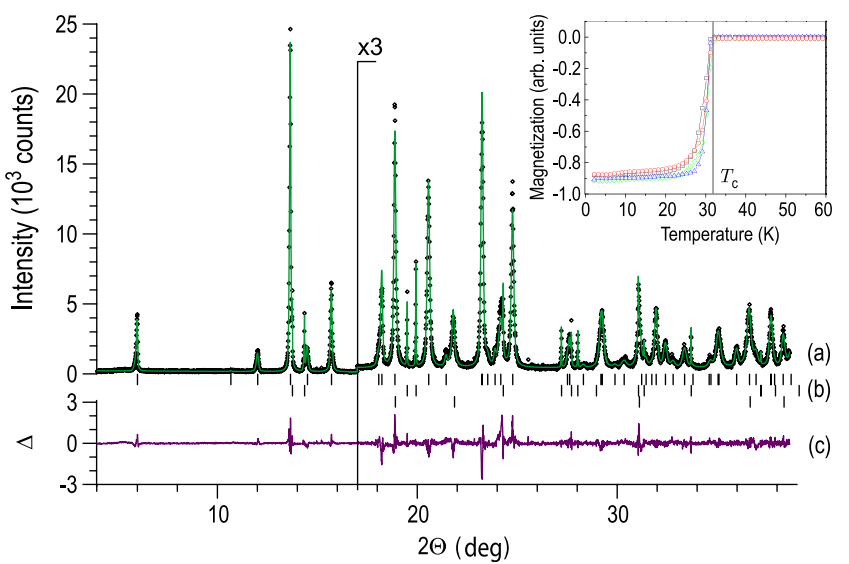

FIG. 1 (color online). Room-temperature XRPD data. (a) Scattered x-ray intensity at $T=300 \mathrm{~K}$ as a function of diffraction angle $2 \Theta(\lambda=0.7 \AA)$ fitted to the tetragonal $I 4 / \mathrm{mmm}$ space group. For $2 \Theta>17^{\circ}$, the plot is enlarged by a factor of 3 . The fit includes a few weight $\%$ of tetragonal $\beta$ tin from the flux as an impurity phase and some reflections of the brass sample holder as indicated by the reflection markers in (b). (c) The difference $\Delta$ between the experimental points and the fitting curve. The inset shows the sharp and reproducible SC transition measured by dc susceptibility on four randomly selected samples.

vealed a sharp SC transition at $T_{c \text {,onset }}=(32 \pm 1) \mathrm{K}$, reproducible among different samples from the same batch. XRPD data confirm that our crystals consist of a single phase fitted well by a tetragonal $I 4 / \mathrm{mmm}$ space group symmetry both at room temperature (see Fig. 1) and at $T=$ $16 \mathrm{~K}$. The room-temperature lattice parameters of the sample, as determined from XRPD by Rietveld refinement using the fundamental parameters approach of TOPAS [16], are $a=b=3.9111(1) \AA$ and $c=13.3392(6) \AA$. Nevertheless, throughout this Letter we will use the orthorhombic notation, inherited from the parent compound.

Figure 2 shows neutron-scattering intensity measured in the vicinity of the $(10 \overline{3})_{\mathrm{O}}$ magnetic Bragg peak $[17,18]$ on a $\sim 30 \mathrm{mg}$ sample with in-plane and out-of plane mosaicities $<1.5^{\circ}$ and $<2.5^{\circ}$, respectively. The final neutron wave vector was set to $k_{f}=1.55 \AA^{-1}$, and a Be filter was used to extinguish contamination from higher-order neutrons. The sample was mounted with the orthorhombic $a$ and $c$ crystallographic directions in the scattering plane in a $15 \mathrm{~T}$ cryomagnet. Panel 2(a) shows $(h 0 \overline{3})_{O}$ scans at three different temperatures. While within the error bar there is no intensity at $100 \mathrm{~K}$, a clear magnetic peak starts to evolve at low temperatures. Panel 2(b) reveals the temperature evolution of the magnetic intensity, which lets us estimate the magnetic transition temperature $T_{m} \approx 70 \mathrm{~K}$.

From the width of the magnetic Bragg peaks, the lower estimate for the correlation length of the AFM phase is $\zeta>$ $100 \AA$. This points to the first major difference in comparison with the underdoped cuprates. In $\mathrm{YBa}_{2} \mathrm{Cu}_{3} \mathrm{O}_{6.45}$, for example, the correlation length of the magnetic order is known to be much smaller, not exceeding $20 \AA$ [19].
Finally, we investigated the effect of a magnetic field $H=13.5 \mathrm{~T}$, applied perpendicularly to the scattering plane and thus in parallel to the FeAs layers. The magnetic intensity was suppressed by $\sim 10 \%$, as shown by solid symbols in Fig. 2(b). This behavior, typical for an antiferromagnet, is again in contrast to the situation in cuprates, where the elastic intensity increases upon application of a magnetic field [20], but is in line with the notion of well-developed magnetic domains with commensurate AFM order.

To gain further insight into the nature of the magnetic ordering - in particular the magnitude of the ordered moment and the magnetic volume fraction-we performed zero-field (ZF) and transverse-field muon-spin rotation ( $\mu$ SR) measurements using $100 \%$ spin polarized muons, which in our setup corresponds to the muon-spin asymmetry of $21 \%$ [21]. The results of our $\mu \mathrm{SR}$ measurements are illustrated by Fig. 3. Panel 3(a) shows the time dependence of the asymmetry, which is a measure for the spin polarization of the muon ensemble. In principle, the oscillation frequency $\nu_{\mathrm{ZF}}$ is determined by the ordered Fe moment $m_{\mathrm{Fe}}$. Since the stopping position of the muon in the lattice is not known precisely, we resort to a comparison with $\mathrm{BaFe}_{2} \mathrm{As}_{2}$, where $m_{\mathrm{Fe}}$ was determined to be $0.4 \mu_{\mathrm{B}}$ [22]. The zero-field frequency for $\mathrm{BaFe}_{2} \mathrm{As}_{2}$ has been established to be $\nu_{\mathrm{ZF}}=28 \mathrm{MHz}$. In comparison, for our sample $\nu_{\mathrm{ZF}}=24.7(5) \mathrm{MHz}$, so we estimate the ordered moment to be only slightly reduced to $\sim 0.35 \mu_{\mathrm{B}}$. This is remarkable, since simultaneously $T_{m}$ is reduced by a factor of 2 from 140 to $70 \mathrm{~K}$.

By applying a weak field of $H=10 \mathrm{mT}$ transverse to the original muon-spin polarization, we can determine the nonmagnetic volume fraction, in which the muons precess around $\mathbf{H}$ conserving the asymmetry, and the magnetically ordered fraction, in which a superposition of external and internal fields depolarizes the beam. Figure 3(b) shows the temperature dependence of the asymmetry in the transverse field. Surprisingly, already at $300 \mathrm{~K}$, we observe a $\sim 21 \%$ loss of asymmetry that might be an indication of a
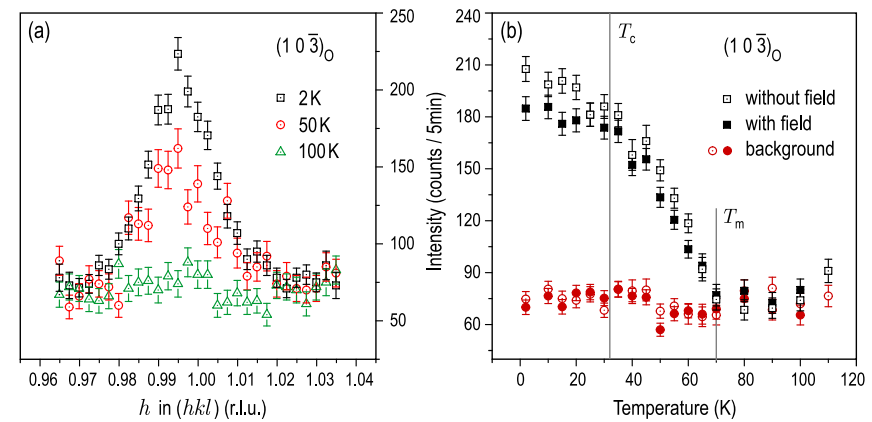

FIG. 2 (color online). Elastic neutron-scattering data measured in the vicinity of the $(10 \overline{3})_{\mathrm{O}}$ magnetic Bragg peak $(\mathrm{O}$ stands for orthorhombic notation). (a) Scans along $(h 0 \overline{3})_{\mathrm{O}}$ at three different temperatures. (b) Temperature evolution of the magnetic intensity. The solid symbols are measured in a magnetic field of 13.5 T applied parallel to the FeAs layers. 

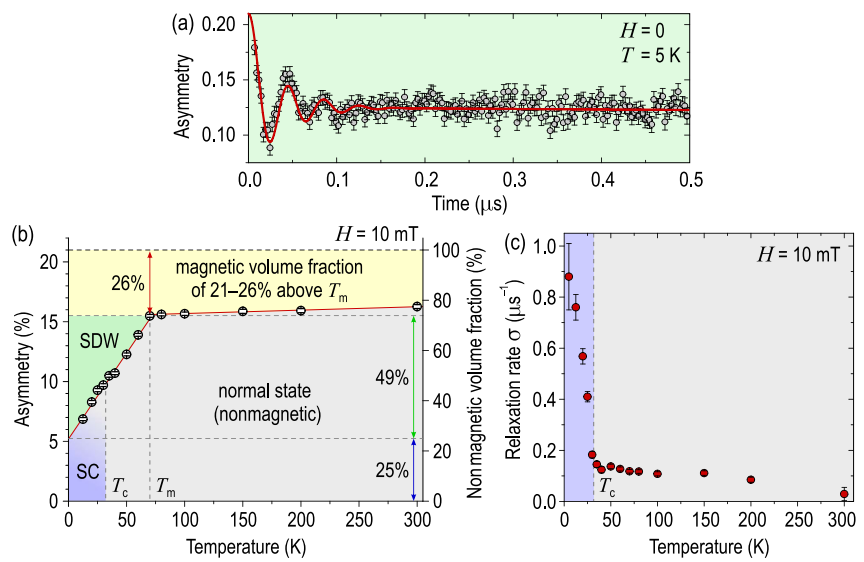

FIG. 3 (color online). $\quad \mu$ SR data. (a) Time dependence of the muon-spin asymmetry in zero field. (b) Temperature dependence of the asymmetry in a weak transverse field, showing coexistence of magnetic and nonmagnetic phases. (c) Temperature dependence of the relaxation rate in a transverse field.

disordered magnetic phase. A straightforward explanation for it would be the presence of a magnetic impurity phase in our sample, such as $\mathrm{Fe}_{2} \mathrm{As}\left(T_{N}=353 \mathrm{~K}\right)$, but such explanation can be ruled out, since XRPD performed on several pieces of samples from the same batch, ground into powder, indicated no presence of parasitic phases, as discussed above. Additionally, angle-resolved photoemission spectroscopy indicates the presence of some kind of density-wave-like order above $T_{m}$ in the same samples, which is weakly temperature dependent [23]. Assuming its magnetic character, it could be speculated that such "hidden" order is possibly responsible for the high-temperature loss of asymmetry observed by $\mu \mathrm{SR}$, which also decreases slightly with temperature above $T_{m}$.

Below $\sim 70 \mathrm{~K}$-the onset temperature of the magnetic intensity at the $(10 \overline{3})_{\mathrm{O}}$ position - the asymmetry further decreases gradually from $15.5 \%$ at $T_{m}$ to $5.2 \%$ at $T \rightarrow 0$, indicating that the volume fraction of the SDW state is $\sim 49 \%$ in the low-temperature limit. The remaining $25 \%$ of the volume phase which remains nonmagnetic at low temperature can be associated with the SC phase. For comparison, in nearly optimally-doped BKFA with $x=0.5$, the low-temperature nonmagnetic volume fraction constitutes almost 50\% [7], in line with the increased $T_{c}=37 \mathrm{~K}$. The $\mathrm{SC}$ volume fraction in our samples was also independently estimated from angle-resolved photoemission spectroscopy [24], which yielded $23 \pm 3 \%$ in agreement with our $\mu \mathrm{SR}$ result.

Note that the decrease in asymmetry below $T_{m}$ is gradual, indicating that we are dealing with a crossover rather than a sharp phase transition. This agrees with the absence of any appreciable anomalies at $T_{m}$ in susceptibility and resistivity measurements.

Finally, we have measured the $\mu \mathrm{SR}$ relaxation rate in the same transverse field. The weak magnetic field penetrates the sample through the AFM islands, creating an inhomogeneous field distribution within the SC phase, which results in a rapid increase of muon depolarization below $T_{c}$, as seen in Fig. 3(c). Thus, the AFM islands act as preformed vortex cores, precluding the formation of an ordered vortex lattice. At $T \rightarrow 0$ the relaxation rate, which in a homogeneous superconductor is expected to be proportional to the superfluid density according to the Uemura relation [25], extrapolates to $\sigma=0.9 \pm 0.1 \mu \mathrm{s}^{-1}$. Surprisingly, this value follows the Uemura relation reasonably well, despite the phase separation. We note that our value of $\sigma$ is higher than that reported for the $x=0.45$ sample in Ref. [26], but still somewhat lower than in the optimally-doped $x=0.5$ sample from Ref. [7].

At this point, we can already conclude that our sample simultaneously exhibits bulk SC with a sharp transition temperature of $32 \mathrm{~K}$ and SDW order with a large correlation length $>100 \AA$, which are spatially separated and change their volume ratio as a function of temperature. This resembles the situation in underdoped cuprates, where SC coexists with a short-range AFM-correlated magnetic state with albeit a strongly reduced ordered magnetic moment [27]. There, however, the magnetic volume fraction seen by $\mu \mathrm{SR}$ is nearly $100 \%$ [27], indicating a more homogeneous coexistence of the two phases. On the other hand, scanning tunneling spectroscopy measurements provide numerous evidence for nanoscale inhomogeneities in the electronic density of states [13]. In contrast to the cuprates, in BKFA we rather observe a mesoscopic phase-separated coexistence [7], as we schematically illustrate in Fig. 4(a), with an ordered moment which is hardly suppressed as compared to the parent compound exhibiting long-range SDW order.

To get a better understanding of the real-space distribution of the magnetically ordered domains, we performed zero-field magnetic force microscopy (MFM) measurements in the SC state on a cleaved surface of a BKFA sample with somewhat reduced $T_{c}$ of $26 \mathrm{~K}$ using an Omicron Cryogenic SFM scanning force microscope supplied with a commercial NanoWorld MFMR magnetic tip possessing a force constant of $\sim 2.8 \mathrm{~N} / \mathrm{m}$ and a resonance frequency of $72 \mathrm{kHz}$. Magnetic contrast was imaged with the lateral resolution $<50 \mathrm{~nm}$ by measuring the frequency shift at a scan height of $10 \mathrm{~nm}$ above the sample surface. As shown in Fig. 4(b), weak static magnetic contrast is clearly seen below $T_{m}$, which would not be expected for a magnetically homogeneous sample. Successive scanning of the same area of the sample confirmed the reproducibility of the magnetic contrast at temperatures below $T_{m}$. The contrast is weakened above $T_{m}$, though does not disappear completely. We associate this contrast with AFM domain boundaries like those sketched in the inset in Fig. 4(a), as the stray field produced by uncompensated magnetic moments at such a boundary is likely to result in a magnetic contrast detectable by MFM. To estimate the characteristic spatial scale of the observed inhomogeneities, we performed a Fourier transform of the MFM signal [see Figs. 4(c) and 4(d)]. The highest-frequency peak in the 

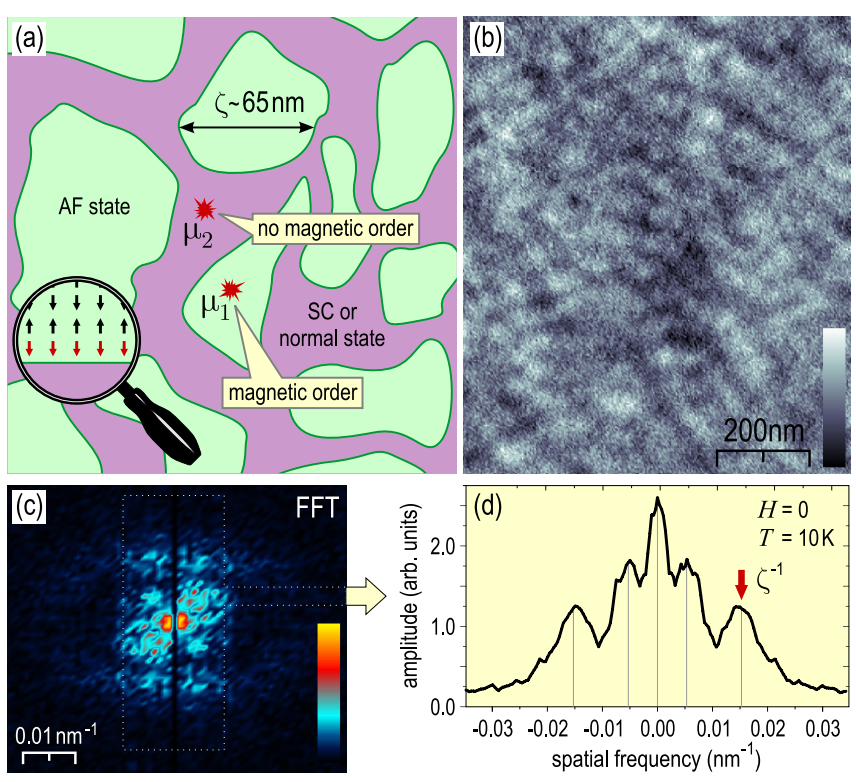

FIG. 4 (color online). (a) Cartoonish representation of the phase-separated coexistence of AFM and SC/normal states. (b) MFM image measured at $10 \mathrm{~K}$ in the absence of external field, revealing weak magnetic contrast on the lateral scale of $\sim 65 \mathrm{~nm}$, as can be estimated from the Fourier-transformed image in panel (c). Panel (d) shows the corresponding spatial frequency profile integrated within the dotted rectangle. The arrow marks the highest-frequency peak in the spectrum, responsible for the $65 \mathrm{~nm}$ modulations.

spectrum corresponds to the characteristic scale of the inhomogeneities of the order of $\zeta=65 \pm 10 \mathrm{~nm}$. A peak corresponding to larger-scale modulations can also be seen in some of the spectra.

To summarize, we have observed mesoscopic phaseseparated coexistence of magnetically ordered and nonmagnetic states on the lateral scale of $65 \pm 10 \mathrm{~nm}$ in a slightly underdoped iron pnictide superconductor, as estimated from MFM imaging in agreement with the $\mu \mathrm{SR}$ measurements. Such electronic phase separation is an intrinsic property of the studied material, as both XRPD and susceptibility measurements indicate the absence of chemical inhomogeneities in the samples. The lateral scale of the phase separation is reminiscent of the situation in superoxygenated $\mathrm{La}_{2} \mathrm{CuO}_{4+\delta}$, where it happens on similar scales of 30-300 nm [12], but is markedly different from the quasihomogeneous nanoscale mixture of electronic phases in most other cuprates [13].

The experimental work was performed at the Swiss spallation source SINQ (RITA-II spectrometer), the Swiss Muon Source, both at the Paul Scherrer Institut (PSI), Villigen, Switzerland, and the X16C beamline at the National Synchrotron Light Source, Brookhaven National Laboratory, USA. MFM measurements were supported by the European Community under the 6th Framework Programme Contract No. 516858: HIPERCHEM. Image processing was done using the WSXM 4.0 software [28]. We acknowledge financial support from DFG in the consortium FOR538, as well as from FCI and BMBF. We also thank P. W. Stephens for the assistance with XRPD measurements and A. Leineweber from MPI-MF for helpful discussions.

*Corresponding author. v.hinkov@fkf.mpg.de

[1] Y. Kamihara et al., J. Am. Chem. Soc. 130, 3296 (2008).

[2] M. Rotter, M. Tegel, and D. Johrendt, Phys. Rev. Lett. 101, 107006 (2008).

[3] I. I. Mazin et al., Phys. Rev. Lett. 101, 057003 (2008); K. Kuroki et al., ibid. 101, 087004 (2008); M. M. Korshunov and I. Eremin, Phys. Rev. B 78, 140509(R) (2008).

[4] H. Luetkens et al., Nature Mater. (in press).

[5] J. Zhao et al., Nature Mater. 7, 953 (2008).

[6] H. Chen et al., Europhys. Lett. 85, 17006 (2009).

[7] T. Goko et al., arXiv:0808.1425.

[8] A. J. Drew et al., Phys. Rev. Lett. 101, 097010 (2008).

[9] J. Wu, P. Phillips, and A. H. Castro Neto, Phys. Rev. Lett. 101, 126401 (2008); Q. Si and E. Abrahams, ibid. 101, 076401 (2008); K. Haule, J. H. Shim, and G. Kotliar, ibid. 100, 226402 (2008); D. J. Singh and M.-H. Du, ibid. 100, 237003 (2008); S. Raghu et al., Phys. Rev. B 77, 220503 (R) (2008).

[10] A. J. Drew et al., Nature Mater. (in press).

[11] E. Dagotto, Science 309, 257 (2005); V. B. Shenoy, D. D. Sarma, and C. N. R. Rao, Chem. Phys. Chem. 7, 2053 (2006).

[12] J. D. Jorgensen et al., Phys. Rev. B 38, 11337 (1988); J. Ryder et al., Physica C (Amsterdam) 173, 9 (1991); A. T. Savici et al., Phys. Rev. B 66, 014524 (2002); H. E. Mohottala et al., Nature Mater. 5, 377 (2006).

[13] S. H. Pan et al., Nature (London) 413, 282 (2001); K. M. Lang et al., ibid. 415, 412 (2002); M. C. Boyer et al., Nature Phys. 3, 802 (2007); A. N. Pasupathy et al., Science 320, 196 (2008).

[14] A. Moreo, S. Yunoki, and E. Dagotto, Science 283, 2034 (1999); M. Fäth et al., ibid. 285, 1540 (1999); J. Burgy et al., Phys. Rev. Lett. 87, 277202 (2001).

[15] G. L. Sun et al., arXiv:0901.2728.

[16] R. W. Cheary, A. A. Coelho, and J. P. Cline, J. Res. Natl. Inst. Stand. Technol. 109, 1 (2004).

[17] Y. Su et al., Phys. Rev. B 79, 064504 (2009).

[18] J. Zhao et al., Phys. Rev. Lett. 101, 167203 (2008).

[19] V. Hinkov et al., Science 319, 597 (2008).

[20] B. Lake et al., Nature (London) 415, 299 (2002); J. Chang et al., Phys. Rev. B 78, 104525 (2008).

[21] J. H. Brewer, Muon Spin Rotation/Relaxation/Resonance, Encyclopedia of Applied Physics Vol. 11 (VCH Publishers, New York, 1995), p. 23.

[22] M. Rotter et al., Phys. Rev. B 78, 020503(R) (2008).

[23] V. B. Zabolotnyy et al., Nature (London) 457, 569 (2009).

[24] D. V. Evtushinsky et al., Phys. Rev. B 79, 054517 (2009).

[25] Y. J. Uemura et al., Phys. Rev. Lett. 62, 2317 (1989).

[26] A. A. Aczel et al., Phys. Rev. B 78, 214503 (2008).

[27] Ch. Niedermayer et al., Phys. Rev. Lett. 80, 3843 (1998).

[28] I. Horcas et al., Rev. Sci. Instrum. 78, 013705 (2007). 